\title{
Model Survivability Complex Object in a Drawing Massive Air Strike
}

\author{
Sergey A. Panov*, \\ Miroslav V. Khalezov and Andrey N. Shmarov \\ Military Training and Research Center of the Air Force \\ "Air Force Academy ft. Professor N.E. Zhukovsky and Y.A. Gagarin» \\ 54a Starykh Bol'shevikov Str., Voronezh, 394064, Russia
}

Received 01.08.2015, received in revised form 21.09.2015, accepted 09.03.2016

The article describes the main provisions of a mathematical model of the survivability of a complex object in a drawing massive air strike, reflecting the process of leaving drums of aviation complexes of the enemy in a given area and to overcome them multifunctional air defense system under cover of objects by means of an integrated (electronic and fire), and taking into account the two-way nature of the conduct EW. The model is based on the representation of these processes in the form of semi-Markov processes described by direct integral-differential equations of the principle of state space extension and application of the Laplace transform.

Keywords: complex object, airfield-based mathematical model, state graph, the transfer function.

Citation: Panov S.A., Khalezov M.V., Shmarov A.N. Model survivability complex object in a drawing massive air strike, J. Sib. Fed. Univ. Eng. technol., 2016, 9(3), 423-434. DOI: 10.17516/1999-494X-2016-9-3-423-434.

\section{Модель живучести сложного объекта}

\section{в условиях нанесения}

\section{массированного авиационного удара}

\author{
С.А. Панов, М.В. Халезов, А.Н. Шмаров \\ ВУНЦ ВВС «ВВА им. проф. Н.Е. Жуковского и Ю.А. Гагарина» \\ Россия, 371600, Воронеж, ул. Старых Большевиков, $54 \mathrm{~A}$
}

В статье приводятся основные положения математической модели живучести сложного объекта в условиях нанесения массированного авиационного удара, отражающей процесс выхода ударных авиационных комплексов противника в заданные районы и преодоления ими многофункичиональной системы ПВО в условиях прикрытия объектов средствами комплексного (радиоэлектронного и огневого) поражения и с учетом двухстороннего характера

(C) Siberian Federal University. All rights reserved

* Corresponding author E-mail address: sergey_panov_1966@mail.ru 
ведения радиоэлектронной борьбы (РЭБ). Модель построена на основе представления рассматриваемых процессов в виде полумарковских процессов, описываемых с помощью прямых интегродифференциальных уравнений, реализации принщипа растирения пространства состояний и применения методов преобразования Лапласа.

Ключевые слова: сложный объект, аэродром базирования, математическая модель, граф состояний, передаточная функция.

\section{Введение}

Опыт локальных войн и вооруженных конфликтов конца XX - начала XXI в. показывает, что одной из важнейших задач, стоящих перед авиацией, является поражение сложных объектов противника с использованием высокоточного авиационного оружия $[1,2]$. К таким сложным объектам могут быть отнесены, например, основные (важнейшие) объекты общевойскового соединения Сухопутных войск, основные элементы авиационных баз и аэродромов наземного базирования авиации, экономические и политические центры, а также многие другие важные объекты инфраструктуры государства. Для эффективного выполнения задачи защиты этих объектов создается специальная группировка средств комплексного (радиоэлектронного и огневого) поражения средств воздушного нападения противника. Обоснование облика, основных технико-тактических характеристик (ТTX), способов и форм боевого применения такой группировки средств комплексного поражения базируется на результатах количественной оценки эффективности нанесения авиационными комплексами удара с использованием высокоточного оружия по сложным объектам в различных оперативно-тактических условиях с учетом двустороннего характера ведения РЭБ. Получение таких количественных оценок невозможно без детального рассмотрения и математического моделирования процесса преодоления авиационными комплексами противника многофункциональной системы прикрытия сложных объектов и последующего нанесения по ним ударов с применением высокоточного оружия. Такие оценки должны проводиться с учетом ожидаемого эффекта от совместного применения средств РЭБ, имитации, маскировки, зенитно-ракетных и зенитно-артиллерийских средств (комплексов) ПВО, используемых для организованного прикрытия сложных объектов. Кроме того, при этом должны учитываться различные тактические приемы и способы радиоэлектронного поражения со стороны противника важнейших радиоэлектронных средств указанной системы, применяемых для прикрытия таких сложных объектов.

В [3, 4] рассмотрены математические модели, отражающие процесс выхода авиационных комплексов противника в районы размещения сложных объектов поражения для нанесения авиационных ударов. Эти модели обеспечивают исследование способов прикрытия сложных объектов на дальних подступах, но не позволяют проводить оценку степени ущерба, наносимого этим объектам авиационной группой, прорвавшей дальние рубежи защиты. Оценка наносимого сложному объекту ущерба дает возможность сделать вывод о его живучести и готовности к выполнению стоящих перед ним задач и в конечном итоге определить номенклатуру средств защиты и их рациональное распределение по рубежам прикрытия. Кроме того, эти модели не позволяют получить оценки вероятностно-временных характеристик системы прикрытия сложного объекта в зависимости от характеристик налета, эффективности авиационных комплексов противника, количества основных элементов сложного объекта и способов их 
защиты. В $[5,6]$ рассмотрены математические модели, отражающие процесс информационного конфликта средств поиска и обнаружения при непосредственной защите объектов от высокоточного авиационного оружия в ближней зоне. Эти модели обеспечивают исследование различных тактических приемов и способов защиты объектов от их обнаружения и последующего поражения высокоточным авиационным оружием. Однако они не позволяют проводить оценку степени ущерба сложным объектам, наносимого высокоточным авиационным оружием, с учетом выхода ударных авиационной группы противника в заданные районы и преодоления ими многофункциональной системы ПВО в условиях прикрытия объектов средствами комплексного (радиоэлектронного и огневого) поражения.

Поэтому в рамках статьи основное внимание будет уделено разработке математического описания процесса нанесения авиацией противника удара по основным элементам сложного объекта, учитывающего эффект от реализации различных тактических приемов и способов применения авиационных комплексов, нанесения ударов высокоточным оружием и их сочетание, с одной стороны, а также с учетом эффекта от совместного использования средств РЭБ, имитации, маскировки, зенитно-ракетных и зенитно-артиллерийских средств (комплексов) ПВО, применяемых для прикрытия сложных объектов в условиях ведения двусторонней РЭБ, - с другой.

В данной статье при выводе основных математических соотношений для примера под сложным объектом будет рассматриваться типовой аэродром наземного базирования авиации $\mathrm{BBC}$, а в качестве цели нанесения противником авиационного удара с применением высокоточного оружия - блокирование аэродрома базирования.

\section{Математическое описание модели процесса поражения сложного объекта высокоточным оружием авиационных комплексов}

Рассмотрим описательную модель, ориентированную на получение объективных исходных данных для построения математической модели процесса поражения основных элементов аэродрома базирования, выход из строя которых исключает взлет авиации и ее дальнейшее применение (блокирование) в течение определенного времени, т. е. отражает живучесть сложного объекта в условиях нанесения массированного авиационного удара. В качестве основных элементов, которые рассматриваются противником как первоочередные цели при блокировании аэродромов базирования, могут выступать: самолеты на стоянках, взлетно-посадочные полосы, рулежные дорожки и укрытия, дальние и ближние приводы посадки, командные пункты и пункты управления полетом, светосигнальные установки (системы) и др. [3]. Соответственно, поражение или вывод из строя какого-либо элемента (или их сочетания) приводит к снижению функциональности аэродрома, увеличению временных затрат на его восстановление и, как следствие, блокированию взлета и посадки авиационных комплексов.

При построении математической модели будем рассматривать многофункциональную систему радиоэлектронного и огневого поражения, создаваемую для прикрытия аэродромов базирования авиации ВВС, которая представляется в виде нескольких зон (рубежей), в каждой из которых могут применяться различные по предназначению средств РЭБ, имитации, маскировки, зенитно-ракетные и зенитно-артиллерийские средства (комплексы) ПВО. Авиационные комплексы противника могут быть поражены только при их нахождении непосред-

$$
-425 \text { - }
$$


ственно в зоне действия зенитно-ракетных и зенитно-артиллерийских средств (комплексов) ПВО, функционирующих в условиях ведения двусторонней РЭБ. Причем время нахождения авиационных комплексов противника в зоне поражения зенитно-ракетных средств (комплексов) многофункциональной системы ПВО $T_{n з}$ является случайной величиной с плотностью распределения $\varphi_{n з}(t)$. Противником для обеспечения действий авиационных комплексов при нанесении высокоточным оружием блокирующих ударов по аэродромам и при поражении средств их прикрытия могут применяться средства имитации, маскировки, разведки и РЭБ. Следовательно, при разработке математической модели будет рассматриваться процесс поражения сложного объекта высокоточным оружием авиационных комплексов противника с учетом преодоления ими многофункциональной системы прикрытия объекта в условиях ведения радиоэлектронного поражения и контррадиоэлектронного поражения важнейших радиоэлектронных средств систем управления войскам и оружием, т. е. ведения двусторонней РЭБ.

Применительно для каждого конкретного типа авиационного комплекса, его вооружения, характеристик выбранных для поражения элементов аэродромов базирования авиации $\mathrm{BBC}$, способов и характеристик прикрытия аэродрома средствами комплексного поражения противником и заданных условий ведения РЭБ, будет реализовываться индивидуальное функционирование авиационного комплекса при нанесении блокирующего удара. Поэтому применительно к построению модели процесса поражения сложного объекта авиационным высокоточным оружием в условиях РЭБ будем рассматривать обобщенную модель функционирования авиационного комплекса при нанесении удара высокоточным оружием по заданным элементам аэродрома в условиях его прикрытия средствами комплексного (радиоэлектронного и огневого) поражения. В ней будет учитываться выполнение авиационным комплексом боевой задачи на следующих этапах: этап взлета; этап полета на малой (предельно малой) высоте (этап выхода в район выполнения боевой задачи); этап доразведки и выхода на заданный элемент объект поражения; этап применения оружия (этап нанесения ударов авиационным комплексом по заданным элементам аэродрома базирования); этап оценки результата нанесения авиационного удара и этап выхода из зоны поражения. При этом необходимо отметить, что авиационный комплекс может выполнять задачу блокирования аэродрома базирования авиации ВВС самостоятельно или совместно с другими авиационными комплексами, также он может осуществлять последовательное нанесение нескольких авиационных ударов с применением высокоточного оружия по каждому из элементов сложного объекта поражения.

С учетом изложенного граф состояний, отражающий функционирование авиационного комплекса при выполнении боевой задачи - поражение сложного объекта высокоточным оружием в условиях РЭБ, на этапах его выхода в район размещения аэродрома базирования и последующего поражения заданных его элементов высокоточным оружием может быть представлен в виде, приведенном на рис. 1.

На рис. 1 приняты следующие обозначения:

$\mathrm{C}_{0}-l$-й авиационный комплекс противника осуществил взлет и получил задачу по нанесению авиационного удара с применением высокоточного оружия для блокирования аэродрома базирования авиации ВBC; 


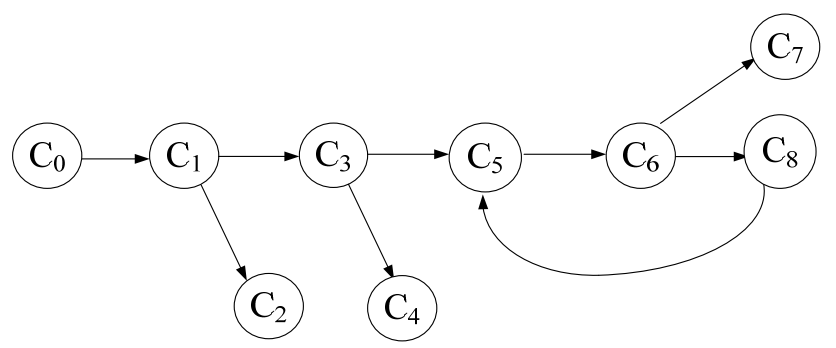

Рис. 1. Граф состояний, отражающий динамику выхода авиационного комплекса в район размещения аэродрома базирования и последующего поражения заданных его элементов одним авиационным комплексом

$\mathrm{C}_{1}-l$-й авиационный комплекс противника вышел в район выполнения боевой задачи и получил целеуказание по объектам поражения авиационным высокоточным оружием - заданным элементам аэродрома базирования;

$\mathrm{C}_{2}-l$-й авиационный комплекс противника не вышел в зону преодоления защитного рубежа (авиационный комплекс уничтожен);

$\mathrm{C}_{3}-l$-й авиационный комплекс противника вышел в зону преодоления защитного рубежа заданного элемента поражения аэродрома базирования;

$\mathrm{C}_{4}-l$-й авиационный комплекс противника не преодолел защитный рубеж (авиационный комплекс уничтожен или не вскрыл объекты поражения - заданные элементы аэродрома базирования);

$\mathrm{C}_{5}-l$-й авиационный комплекс противника преодолел защитный рубеж и вскрыл заданные объекты поражения - элементы аэродрома базирования;

$\mathrm{C}_{6}-l$-й авиационный комплекс противника нанес с применением высокоточного оружия $\mathrm{n}$-й удар по заданным элементам поражения аэродрома базирования;

$\mathrm{C}_{7}$ - аэродром блокирован в результате нанесения n-го удара высокоточным оружием $l$-м авиационного комплекса противника;

$\mathrm{C}_{8}$ - аэродром не блокирован в результате нанесения n-го удара высокоточным оружием $l-$ го авиационного комплекса противника.

Если предположить, что для ряда этапов (отражаемых переходами из состояния $\mathrm{C}_{0}$ в состояние $\mathrm{C}_{1}$, из состояния $\mathrm{C}_{5}$ в состояние $\mathrm{C}_{6}$, и из состояния $\mathrm{C}_{8}$ в состояние $\mathrm{C}_{5}$ ) на интервалах времени выполнения боевых задач авиационными комплексами возможностью их поражения можно пренебречь, то влияние средств комплексного (радиоэлектронного и огневого) поражения на эффективность защиты сложного объекта в различных условиях будет описываться изменением характеристик закона распределения времени перехода системы между состояниями и характеризоваться только применением различной номенклатуры и количества средств (комплексов) РЭБ, имитации и маскировки, а также реализуемых тактических приемов и способов их применения при прикрытии объектов. Для определения этих характеристик осуществляется расширение пространства состояний рассматриваемого процесса за счет введения дополнительных промежуточных состояний, отражающих выполнение задач авиационным комплексом в измененных условиях $[5,7]$. Для примера дополнительно рассмотрим условия постановки преднамеренных помех системе выдачи целеуказания. Тогда для описания процесса перехода 
из состояния $\mathrm{C}_{0}$ в состояние $\mathrm{C}_{1}$ введем дополнительное состояние $\mathrm{C}_{01}$ - преднамеренные помехи поставлены до момента времени выхода авиационного комплекса противника в район выполнения боевой задачи и получения целеуказания по объектам поражения. Граф состояний, иллюстрирующий расширение пространства состояний для перехода процесса между состояниями $\mathrm{C}_{0}, \mathrm{C}_{1}$, представлен на рис. 2.

$\mathrm{B}$ этом случае процесс перехода из состояния $\mathrm{C}_{0}$ в состояние $\mathrm{C}_{1}$ с физической точки зрения отражает процесс получения авиационным комплексом целеуказания по объектам поражения до момента времени постановки преднамеренных помех. При этом процесс перехода из состояния $\mathrm{C}_{0}$ в состояние $\mathrm{C}_{01}$ будет отображать противоположное событие. Переход процесса в состояние $\mathrm{C}_{1}$ из состояния $\mathrm{C}_{01}$ отражает процесс получения авиационным комплексом целеуказания по объектам поражения в условиях постановки преднамеренных помех. Применительно к рассмотренным условиям, как показано в [5], определяются характеристики обобщенного закона распределения времени перехода процесса из состояния $\mathrm{C}_{0}$ в состояние $\mathrm{C}_{1}$.

Для остальных этапов (отражаемых переходами в состояния $\mathrm{C}_{3}, \mathrm{C}_{5}$ и $\mathrm{C}_{7}$ ) на интервалах времени выполнения боевых задач авиационными комплексами будет учитываться совместное применение различных по предназначению средств (комплексов) РЭБ, имитации, маскировки, зенитно-ракетных и зенитно-артиллерийских средств (комплексов) ПВО, функционирующих в условиях ведения двусторонней РЭБ. Определение характеристик обобщенных законов распределения времени перехода процесса в указанные состояния в условиях применения только средств (комплексов) РЭБ, имитации и маскировки (т. е. в условиях отсутствия возможностей поражения авиационных комплексов средствами ПВО) осуществляется аналогично рассмотренному выше. В целом граф состояний, отражающий процесс выполнения авиационным комплексом задач в условиях совместного применения средств (комплексов) РЭБ, имитации, маскировки и ПВО, схематично представлен на рис. 3.

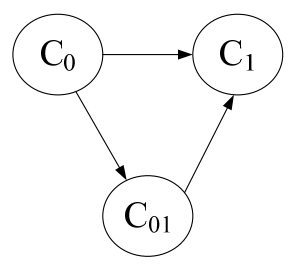

Рис. 2. Граф, отражающий процесс расширения пространства состояний

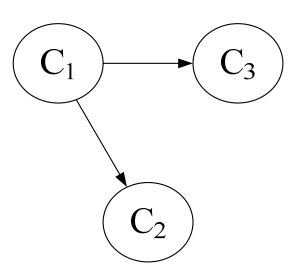

Рис. 3. Граф, отражающий процесс выполнения авиационным комплексом задач в условиях совместного применения средств (комплексов) РЭБ, имитации, маскировки и ПВО 
Так как авиационные комплексы могут быть поражены только во время их нахождения в зоне поражения зенитно-ракетных или зенитно-артиллерийских средств (комплексов) ПВО, процесс перехода из состояния $\mathrm{C}_{1}$ в состояния $\mathrm{C}_{2}$ и $\mathrm{C}_{3}$ можно описать в виде конфликта «самолет - средство (комплекс) ПВО». Тогда с учетом рекомендаций, изложенных в $[3,5,7]$, применительно к условиям протекания такого конфликта в условиях ведения двусторонней РЭБ вероятность того, что $l$-й авиационный комплекс уничтожен (не вышел в зону преодоления защитного рубежа заданного элемента поражения аэродрома базирования) на интервале времени $[t, \mathrm{t}+\mathrm{dt}]$, может быть определена как

$$
\tilde{P}_{c_{l}}=P_{n c_{l}} \cdot \varphi_{n c_{l}}(t) \cdot\left[1-F_{n 3}(t)\right],
$$

где $\tilde{P}_{c_{l}}$ - вероятность поражения $l$-го авиационного комплекса противника на интервале времени $[\mathrm{t}, \mathrm{t}+\mathrm{dt}]$ до входа в зону преодоления защитного рубежа заданного элемента - объекта поражения аэродрома базирования в условиях ведения двусторонней РЭБ; $P_{n c}-$ полигонная вероятность поражения авиационного комплекса противника средствами (комплексами) ПВО, размещенными до входа в зону преодоления защитного рубежа заданного элемента аэродрома в условиях ведения двусторонней РЭБ; $\varphi_{n c_{1}}(t)$ - плотность вероятности времени поражения авиационных комплексов противника зенитно-ракетными средствами (комплексами) ПВО; $F_{n 3}(t)-$ вероятность поражения зенитно-ракетных и зенитно-артиллерийских средств (комплексов), размещенных в зоне ПВО до входа в зону преодоления защитного рубежа заданного элемента аэродрома базирования в условиях ведения двусторонней РЭБ.

Вероятность поражения авиационного комплекса противника за некоторое время $F_{n 3_{1}}$ до входа в зону преодоления защитного рубежа заданного элемента - объекта поражения аэродрома базирования авиации ВВС в условиях ведения двусторонней РЭБ:

$$
P_{c_{l}}^{y}\left(T_{3 n_{l}}\right)=P_{n c_{l}} \cdot \int_{0}^{T_{3 n_{l}}} \varphi_{n c_{l}}(\tau) \cdot\left[1-F_{n 3}(\tau)\right] \cdot d \tau .
$$

Задаваясь законом распределения времени нахождения $l$-го авиационного комплекса противника в зоне его поражения зенитно-ракетными средствами (комплексами), размещенными в зоне ПВО (до входа в зону преодоления защитного рубежа заданного объекта поражения) в условиях ведения двусторонней РЭБ $f_{3 n_{1}}\left(t_{3 n_{1}}\right)$, определим среднюю вероятность поражения авиационного комплекса в условиях ведения двусторонней РЭБ:

$$
\bar{P}_{c_{l}}(t)=\int_{0}^{\infty} P_{n c_{l}}^{y}\left(t_{3 n_{l}}\right) \cdot f_{3 n_{l}}\left(t_{3 n_{l}}\right) \cdot d t_{3 n_{l}}
$$

В математической модели соотношения (1),..,(3), по существу, отражают основные физические процессы преодоления авиационными комплексами противника многофункциональной системы прикрытия и определяют возможность его выхода в район нанесения блокирующего удара по основным элементам аэродромов базирования авиации ВВС с учетом их имитации и маскировки, прикрытия зенитно-ракетными средствами (комплексами) ПВО и РЭБ.

В целом для графа состояний, приведенного на рис. 1, в соответствии с рекомендациями, изложенными в [5-7], можно строго определить передаточные звенья, соединенные в соответствии с дугами графа, которые будут отражать динамику выхода авиационного комплекса

$$
-429-
$$


в район размещения аэродрома базирования и поражение заданных его элементов в виде передаточных звеньев (рис. 4).

Передаточная функция $H_{u}(S)$, соответствующая переходу точка $(\mathbf{A})$ - точка $(\mathbf{B})$, может быть определена из уравнения

$$
H_{u}(s)=\frac{H_{56}(s)}{1-H_{56}(s) \cdot H_{68}(s) \cdot H_{85}(s)} .
$$

Тогда общая передаточная функция

$$
\begin{aligned}
& H_{\Sigma}(s)=H_{01}(s) \cdot H_{13}(s) \cdot H_{35}(s) \cdot H_{u}(s) \cdot H_{67}(s)= \\
& =H_{01}(s) \cdot H_{13}(s) \cdot H_{35}(s) \cdot \frac{H_{56}(s)}{1-H_{56}(s) \cdot H_{68}(s) \cdot H_{85}(s)} H_{67}(s) .
\end{aligned}
$$

Переход точка (А) - точка (В) (рис. 4) с физической точки зрения соответствует тому, что после применения авиационного высокоточного оружия (пуска управляемой ракеты, сброса авиабомбы) цель (заданный элемент поражения аэродрома базирования) оказалась не поражена, тогда авиационный комплекс осуществляет повторный заход на цель и наносит следующий удар.

Для вероятности нахождения в состоянии $\mathrm{C}_{7}$ (аэродром блокирован) к некоторому моменту времени $t$ с учетом нанесения $l$-м авиационным комплексом противника по заданному элементу поражения аэродрома базирования $\mathrm{n}$ авиационных ударов, определяемых как количество циклов (точка $\mathbf{A}$ - точка $\mathbf{B}$ - точка $\mathbf{A}$ ), можно записать [3, 5]:

$$
\tilde{P}_{7}(n, t)=L_{s}^{-1}\left\{H_{01}(s) \cdot H_{13}(s) \cdot H_{35}(s) \cdot H_{56}(s) \cdot\left[H_{56}(s) \cdot H_{68}(s) \cdot H_{85}(s)\right]^{n} \cdot H_{67}(s)\right\},
$$

где $L_{S}^{-1}$ - оператор обратного преобразования Лапласа.

Предположим, что:

$$
\begin{aligned}
& H_{01}(s) \cdot H_{13}(s) \cdot H_{35}(s)=\bar{P}_{l} \cdot \exp ^{-s \bar{T}_{05}} \\
& H_{56}(s) \cdot H_{67}(s)=\bar{P}_{n l} \cdot \exp ^{-s \bar{T}_{57}} \\
& H_{56}(s) \cdot H_{68}(s) \cdot H_{85}(s)=\bar{P}_{n l} \frac{\Lambda_{55}}{\Lambda_{55}+S}
\end{aligned}
$$

где $\bar{P}_{l}$ - средняя вероятность выхода в район выполнения боевой задачи и зону преодоления защитного рубежа аэродрома; $\bar{P}_{n l}-$ средняя вероятность выхода $l$-го авиационного комплекса

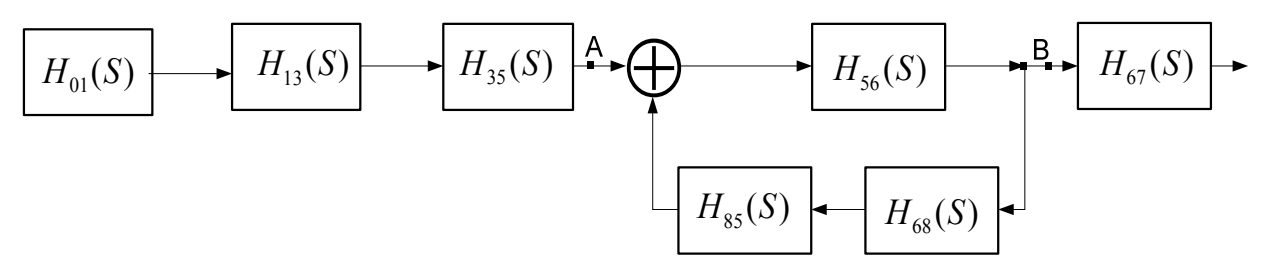

Рис. 4. Представление графа состояний, отражающего динамику выхода авиационного комплекса в район размещения аэродрома базирования и последующего поражения заданных его элементов высокоточным оружием авиационных комплексом, в виде передаточных звеньев 
$(l=1, \ldots, L)$ к рубежу применения оружия по заданному элементу поражения аэродрома базирования в $\mathrm{n}$-м ударе $(\mathrm{n}=1, \ldots, \mathrm{N}) ; S$ - оператор Лапласа; $\bar{T}_{55}$ - средний интервал времени между авиационными ударами, наносимыми $l$-м авиационным комплексом противника по заданному элементу поражения аэродрома базирования; $\Lambda_{55}=1 / \bar{T}_{55}-$ интенсивность нанесения ударов $l$-м авиационным комплексом противника по заданному элементу поражения аэродрома базирования.

Тогда выражение (6) можно записать в виде

$$
\tilde{P}_{7}(n, t)=L_{s}^{-1}\left\{\left[\frac{\bar{P}_{n l} \cdot \Lambda_{55}}{\Lambda_{55}+S}\right]^{n} \overline{P_{l}} \cdot \bar{P}_{n l} \cdot \exp ^{-s\left(\bar{T}_{05}+\bar{T}_{57}\right)}\right\},
$$

где $\bar{T}_{05}$ - среднее время получения задачи (целеуказания) и выхода авиационного комплекса к рубежу применения оружия по заданному элементу поражения сложного объекта (элементу аэродрома базирования); $\bar{T}_{57}$ - среднее время, затрачиваемое на нанесение авиационным комплексом одного удара по заданному элементу поражения сложного объекта для блокирования аэродрома базирования авиации ВВС.

Обозначив $T_{\Sigma}=\bar{T}_{05}+\bar{T}_{57}$ и выполняя операцию обратного преобразования Лапласа, находим

$$
P_{7}(n, t)=\frac{\Lambda_{55}^{n} \cdot P_{n l}^{n}}{(n-1)}\left[t-T_{\Sigma}\right]^{(n-1)} \cdot \exp ^{-\Lambda_{55}\left(t-T_{\Sigma}\right)}\left[t-T_{\Sigma}\right] \cdot \overline{P_{l}} \cdot \bar{P}_{n l},
$$

где $P_{n l}^{n}$ - вероятность применения $l$-м авиационным комплексом высокоточного оружия по заданному элементу поражения сложного объекта в ходе нанесения $n$ ударов.

В этом случае вероятность поражения к-го элемента аэродрома базирования высокоточным оружием $l$-го авиационного комплекса к моменту времени $t$ при нанесении по нему $n$ ударов может быть найдена как

$$
P_{7 k}(n, t)=\frac{\Lambda_{55} \cdot P_{n l}^{n} \cdot \bar{P}_{l}}{(n-1) !} \int_{0}^{t}\left[\Lambda_{55} u^{\prime}\right]^{(n-1)} \exp ^{-\left[\Lambda_{55} u^{\prime}\right]} d u^{\prime}
$$

где $u^{\prime}=t-T_{n \Sigma}$.

Очевидно, что аэродром базирования авиации ВВС может быть блокирован только после нанесения хотя бы одного $(n=1)$ удара авиационным высокоточным оружием по его $\kappa$-му элементу, т. е., $t \geq \bar{T}_{05}+\bar{T}_{57}$. Кроме того, может быть определено и максимальное время нанесения $n$ ударов по $\kappa$-му элементу аэродрома базирования авиации ВВС при его блокировании $T_{n \Sigma}=\bar{T}_{05}+\bar{T}_{n, 57}$. Тогда, обозначив $\gamma_{l}^{n}=P_{n l}^{n} \cdot \bar{P}_{l}$, выражение (9) можно преобразовать к виду

$$
P_{7 k}(n, t)=\left\{\begin{array}{ll}
0, & t<\overline{T_{05}}+\overline{T_{57}} \\
\gamma_{l}^{n} \cdot \frac{\Lambda_{55}}{(n-1) !} \int_{0}^{t}\left[\Lambda_{55} \cdot u^{\prime}\right]^{(n-1)} \cdot e^{-\left[\Lambda_{55} \cdot u^{\prime}\right]} d u^{\prime}, \quad t \geq \overline{T_{05}}+\overline{T_{57}}
\end{array} .\right.
$$

Введя переменные $\Lambda_{55} u^{\prime}=u, \quad d u^{\prime}=\frac{1}{\Lambda_{55}} d u$, выражение (10) можно преобразовать к виду 


$$
P_{7 k}(n, t)=\left\{\begin{array}{ll}
0, & t<\overline{T_{05}}+\overline{T_{57}} \\
\frac{\gamma_{l}^{n}}{(n-1) !} \int_{0}^{u} u^{(n-1)} \cdot \exp ^{-u} d u, & t \geq \overline{T_{05}}+\overline{T_{57}}
\end{array} .\right.
$$

Для интервала времени $t \geq \bar{T}_{05}+\bar{T}_{57}$, выражение (11) может быть сведено к табличному интегралу [8]:

$$
\int_{0}^{u} x^{n} \cdot \exp ^{-\mu x} d x=\frac{n !}{\mu^{(n+1)}}-\exp ^{-u \mu} \sum_{k=0}^{n} \frac{n !}{k !} \cdot \frac{u^{k}}{\mu^{(n-k+1)}}
$$

Тогда, осуществляя интегрирование для интервала времени $t \geq \bar{T}_{05}+\bar{T}_{57}$ и используя обратную подстановку переменных, получим выражение для оценки вероятности поражения $l$-м авиационным комплексом противника $к$-го объекта аэродрома базирования авиации ВВС при его блокировании за время $t \geq \bar{T}_{05}+\bar{T}_{n, 57}$ :

$$
\begin{aligned}
& P_{l}\left(n_{k}, t\right)= \begin{cases}0, & t<\bar{T}_{05}+\bar{T}_{n, 57} \\
P_{n l}^{n} \cdot \bar{P}_{l}\left[1-\exp ^{-u} \cdot \sum_{k=0}^{n-1} \frac{u^{k}}{k !}\right], & t \geq \bar{T}_{05}+\bar{T}_{n, 57}\end{cases}
\end{aligned}
$$

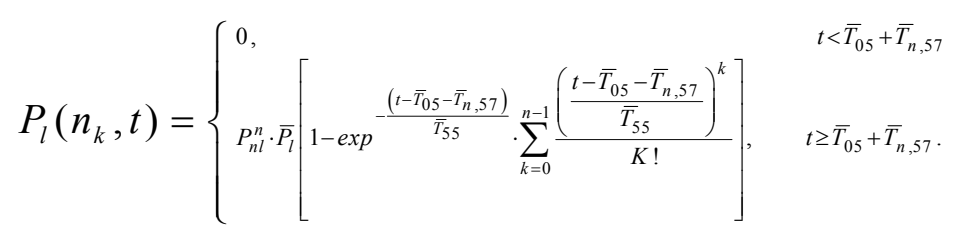

Предположим, что для блокирования аэродрома базирования авиации ВВС на заданное время $T_{\text {б. }}$ необходимо поразить пять его элементов $(\kappa=1, \ldots, 5)$. Для поражения каждого из элементов выделен наряд средств - $l$ авиационных комплексов $(l=1, \ldots, L)$, которые последовательно наносят удары высокоточным оружием до их поражения. Полагая, что процессы поражения элементов аэродрома базирования авиации независимые, вероятность блокирования аэродрома можно определить как

$$
P_{\sigma, l}\left(T_{\sigma, l}\right)=\prod_{k=1}^{5}\left\{1-\prod_{n_{k}=1}^{N} \prod_{l=1}^{L}\left[1-P_{l}\left(n_{k}, t\right)\right]\right\} .
$$

Полученные значения вероятности блокирования аэродрома характеризуют живучесть сложного объекта в условиях нанесения противником массированного авиационного удара и позволяют проводить количественную оценку степени ущерба, наносимого его высокоточным авиационным оружием, с учетом выхода авиационной группы в заданные районы и преодоления ими многофункциональной системы ПВО в условиях ведения двухсторонней РЭБ.

\section{Заключение}

В статье представлены результаты разработки математической модели процесса поражения высокоточным авиационным оружием противника сложного объекта в условиях его прикрытия средствами комплексного (радиоэлектронного и огневого) поражения с учетом двухстороннего характера ведения РЭБ. 
Модель построена на основе представления рассматриваемых процессов в виде полумарковских процессов, их описания как конфликтно обусловленных процессов с помощью прямых интегродифференциальных уравнений, реализации принципа расширения пространства состояний и применения методов преобразования Лапласа. Такое построение модели позволило с требуемой для моделирования детальностью учесть реализацию противником различных тактических приемов и способов применения авиационных комплексов и нанесения ударов высокоточным оружием (или их сочетание). С другой стороны, это позволило учесть влияние совместного использования средств РЭБ, имитации, маскировки, зенитно-ракетных и зенитноартиллерийских средств (комплексов) ПВО, применяемых для прикрытия сложных объектов в условиях ведения двусторонней РЭБ. Предложенная модель дает возможность проводить исследования по оценке эффективности защиты сложного объекта от авиационных ударов противника в условиях РЭБ и на этой основе обосновывать облик, основные тактико-технические характеристики и формы боевого применения средств комплексного (радиоэлектронного и огневого) поражения.

\section{Список литературы}

[1] Ноговицын А.А. Опыт войн и способы боевого применения ВВС и войск ПВО в современных операциях. Военная мысль, 2005, № 6. [Nogovitsin A.A. The experience of war and ways to combat the use of the air force and air defense forces in modern operations. The military thought, 2005, №6 (in Russian)]

[2] Бородин А.А., Букша С.Н., Зибров Г.В. Оперативно-тактические требования к обеспечению живучести базирования авиации в операциях начального периода войны. Вестник ВАИУ, № 2(13). Воронеж: 2011, С. 11-15. [Borodin A.A., Buksha S.N., Zibrov G.V. Operational and tactical requirements to ensure the survivability of aircraft based in the initial period of war operations. Journal of Voronezh Aviation Engineering Institute № 2 (13), Voronezh, 11-15 (in Russian)]

[3] Козирацкий Ю.Л., Панов С.А., Халезов М.В. Модель процесса преодоления авиационными комплексами противника многофункциональной системы ПВО в условиях РЭБ. Вестник ВУНЦ ВВС «ВВА», № 2(13). Воронеж: 2014, C. 116-122. [Koziratsky J.L., Panov S.A., Khalezov M.V. The process model of overcoming the enemy's aviation complex multifunctional air defense system in the conditions of electronic warfare. Journal of Military Training and Research Center of the Air Force «Air Force Academy» № 2(13). Voronezh, 2014, 116-122 (in Russian)]

[4] Козирацкий Ю.Л., Панов С.А., Халезов М.В. Модель процесса поражения основных элементов авиационных баз и аэродромов базирования. Вестник ВАИУ, № 2(16). Воронеж: 2013, C. 28-33. [Koziratsky J.L., Panov S.A., Khalezov M.V. Process Model defeat the basic elements of air bases and airfields home. Journal of Voronezh Aviation Engineering Institute № 2(16) Voronezh, 2013, 28-33 (in Russian)]

[5] Козирацкий Ю.Л., Модель информационного конфликта средств поиска и обнаружения. Монография / под ред. Ю.Л. Козирацкого. М.: Радиотехника, 2013. 232 с. [Koziratsky J.L. Model information conflict search and discovery tools. Moscow: Radiotechnics, 2013. 232 (in Russian)]

[6] Ионов С.В., Паринов М.Л., Чернухо И.И. Модель процесса местоопределения источников радиоизлучения с использованием перспективных антенных систем. Вестник ВАИУ, № 1(12). Воронеж: 2012, C. 200-204. [Ionov S.V, Parinov M.L., Chernukho I.I. Process Model,

$$
-433-
$$


positioning of radio emission sources using advanced antenna systems. Journal of Voronezh Aviation Engineering Institute № 1(12), Voronezh, 2012, 200-204 (in Russian)]

[7] Козирацкий Ю.Л., Донцов А.А., Кущев С.С., Чернухо И.И. Модель конфликтного взаимодействия систем управления противоборствующих сторон в условиях преднамеренных помех. М.: Радиотехника. 2012. № 5. C. 56-61. [Koziratsky Y.L., Dontsov A.A., Kushchev S.S., Chernukho I.I. The model of conflict interaction of control systems of the opposing sides in a of intentional interference. Moscow: Radiotechnics. 2012. № 5. 56-61. (in Russian)]

[8] Градштейн И.С., Рыжик И.М. Таблищь интегралов, сумм, рядов и произведений. М.:, Физматгиз, 1963,-324 c. [Gradshtein I.S., Ryzhikv I.M. Tables of integrals, sums, series and products. Moscow: Fizmatgiz, 1963, 324 (in Russian)] 